\title{
Robust Inverse Dynamic Control of a Maneuvering Smart Flexible Satellite with Piezoelectric Layers
}

\author{
Mohammad Azadi \\ Department of Mechanical Engineering, Marvdasht Branch, Islamic Azad University, Marvdasht, I.R.Iran.
}

\section{Emad Azadi}

Department of Mechanical Engineering, Faculty of Shahid Bahonar, Shiraz Branch, Technical and Vocational University (TVU), Shiraz, Iran.

\author{
S. Ahmad Fazelzadeh \\ School of Mechanical Engineering, Shiraz University, Shiraz, I.R.Iran.
}

(Received 21 January 2015; accepted 25 August 2015)

In this paper, a satellite with two flexible appendages and a central hub is considered. The piezoelectric layers are attached to both side of the appendages and used as actuators. The governing equations of motion are derived based on Lagrange method. Using Rayleigh-Ritz technique ordinary differential equations of motion are obtained. A robust inverse dynamic control is applied to the system to not only control the three axes manoeuvre of the satellite but also suppress the vibrations of the flexible appendages. Finally, the system is simulated and simulation results show good performance of this controller.

\section{INTRODUCTION}

The large angle attitude rotation of a spacecraft is one of its major desirable advantages. The modern flexible spacecrafts need to re-point and track manoeuvres. The dynamics of the spacecraft with large rotational manoeuvre is time varying and nonlinear. Moreover, the manoeuvring introduces certain levels of vibration to flexible appendages.

Most studies on spacecrafts consist of the manoeuvre control of spacecraft around single axis rotation. Song and Kotejoshyer ${ }^{1}$ studied vibration reduction of flexible structures during slew operations such as attitude control of spacecraft with large solar panels. Ge et al. ${ }^{2}$ applied an energy-based robust control approach for a flexible spacecraft attitude regulating control, as well as the vibration suppression of the flexible appendages. A robust adaptive sliding mode control was presented by $\mathrm{Hu}^{3}$ to control the attitude manoeuvring of a flexible spacecraft, and linear quadratic regulator (LQR) was utilized for suppressing the induced vibration in the appendages. Erdong and Zhaowei ${ }^{4}$ investigated an attitude regulation control problem for a flexible spacecraft. Azadi et al.5,6 studied the hybrid controller to suppress the appendage vibrations and manoeuvring control of a one axis rotating flexible satellite.

Recently, some researchers have benefitted from using smart materials like piezoelectrics for sensors/actuators. $\mathrm{Hu}$ and $\mathrm{Ma}{ }^{7}$ using the theory of variable structure control, presented an approach to reduce the vibration of the flexible spacecraft during attitude manoeuvre. $\mathrm{Hu}^{8}$ also proposed a composite control scheme for a single angle rotational spacecraft with bounded piezoelectric material on its flexible appendages, satisfying the actuator constraints in the presence of mismatched perturbation. Neto et al. ${ }^{9}$ presented a general methodology for active vibration control of high-speed flexible linkage mechanisms with piezoelectric actuators and sensors. Further, equations of motion for flexible multi-body systems and for the relevant finite elements used in the modelling of piezoelectric materials were introduced. Sales et al. ${ }^{10}$ considered the appli- cation of shunted piezoelectric transducers to control the vibrations of a simple satellite composed of a hub, a reaction wheel, which is a device traditionally used for active attitude control, and two identical flexible panels, which contain piezoelectric patches symmetrically bonded to their surfaces.

Some scientists have studied three axes rotational manoeuvre control of flexible satellites. $\mathrm{Hu}^{11}$ applied a semi-globally input-to-state control technique to a flexible spacecraft attitude manoeuvre in the presence of parameter uncertainties and external disturbances. A fault- tolerant adaptive backstepping sliding mode control scheme was developed for flexible spacecraft attitude manoeuvring using redundant reaction wheels by Jiang et al. ${ }^{12}$ Xin and Pan $^{13}$ investigated a nonlinear optimal control to approach and align a spacecraft to a target. Azadi et al. ${ }^{14}$ applied an adaptive robust controller to control the three axes manoeuvre of a satellite while suppressing the appendages vibrations using piezoelectric actuators.

In some studies, the satellite has been considered as a rigid body and the flexibility effects have been neglected. Verbin and Lappas ${ }^{15}$ presented a new attitude control method for agile rigid spacecrafts, which was based on combining single gimbal control moment gyros together with reaction wheels. The method was expected to suit remote sensing spacecrafts that are required to perform multiple rapid retargeting of their line of sight. They ${ }^{16}$ also presented a nonlinear state feedback design approach for large rotational manoeuvring of a rigid satellite with reaction wheels. Huang et al. ${ }^{17}$ studied the dynamic and control hovering of a rigid spacecraft. The dynamical model, which they considered, was built to describe the orbital motion of the spacecraft, and a control scheme was derived for different hovering configuration.

In this study a robust inverse dynamic control scheme is used to control the three axes manoeuvre of a satellite and suppress the vibrations of the appendages. The satellite is considered as a central hub with two flexible appendages. The piezoelectric layers are attached to both sides of the appendages as actuators. Each appendage is considered as an Euler-Bernoulli 


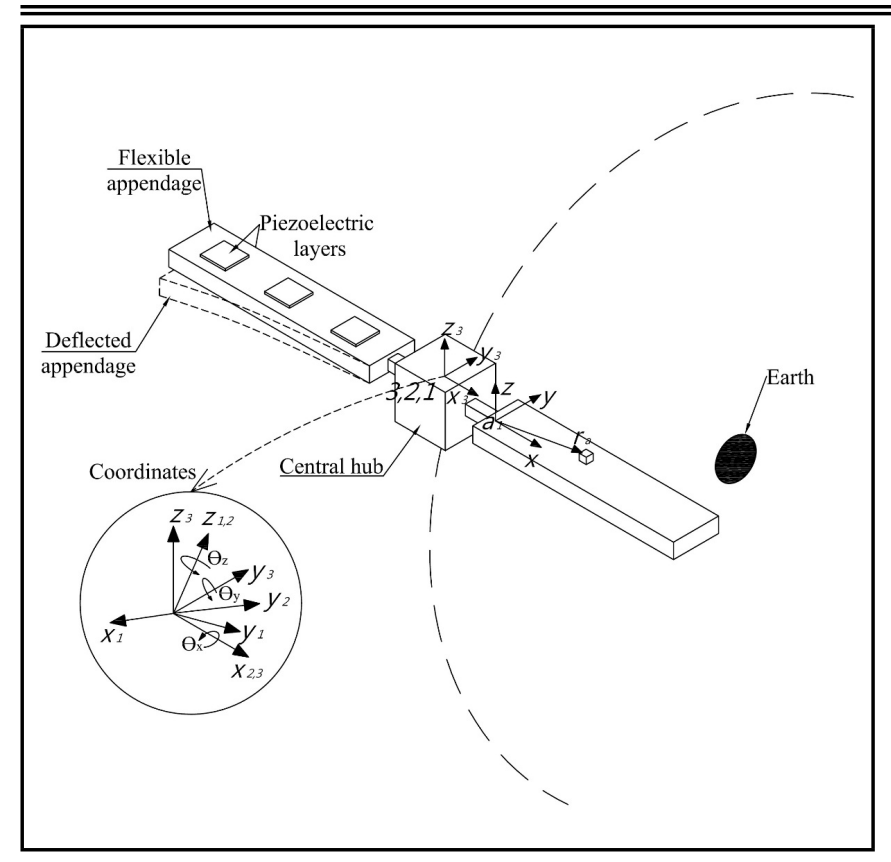

Figure 1. Schematic of the satellite with coordinate systems.

beam. The nonlinear governing equations of motion are derived and the system is simulated to measure the controller robustness.

\section{SYSTEM DYNAMICS}

Consider a satellite with flexible appendages, which is shown in Fig. 1. This satellite moves in the space with constant velocity and manoeuvres around three axes. The appendages of this satellite are considered as Euler-Bernoulli beams, which have width $b$, thickness $h$, length $L$, density $b$, and Young's modulus $E$, and the piezoelectric layers are attached to them. Each piezoelectric layer has thickness $h_{p}$, density $\rho_{p}$, Young's modulus $E_{p}$, and the equivalent piezoelectric coefficient $e_{31}$. $w_{i}(x, t)$ represents the lateral deflection of the ith satellite appendage. Knowing the kinetic and potential energy of the system is necessary for using Lagrange method to derive the governing equations of motion of the system.

The kinetic energies of the nth appendage, piezoelectric layers, and hub are as:

$$
\begin{aligned}
T_{a} & =\frac{1}{2} \sum_{i=1}^{2} \int_{\text {appendage }_{i}} \operatorname{trace}\left\{\dot{r}_{R}\left(\dot{r}_{R}\right)^{T}\right\} d m ; \\
T_{p} & =\frac{1}{2} \sum_{i=1}^{2} \sum_{n=1}^{N_{i}} \int_{P Z T_{n}} \operatorname{trace}\left\{\dot{r}_{R}\left(\dot{r}_{R}\right)^{T}\right\} d m ; \\
T_{h} & =\frac{1}{2} \int_{\text {hub }} \operatorname{trace}\left\{\dot{r}_{R}\left(\dot{r}_{R}\right)^{T}\right\} d m ;
\end{aligned}
$$

where $\dot{r}_{R}$ is the velocity vector of an element with respect to reference frame. $N_{i}$ is the number of piezoelectric layers in $i$ th appendage. The potential energies of the appendages and piezoelectric layers are:

$$
U_{a}=\frac{1}{2} \sum_{i=1}^{2} \int_{\text {appendage }_{i}} E z^{2}\left(\frac{\partial^{2} w_{i}}{\partial x^{2}}\right)^{2} d v
$$

$$
\begin{gathered}
U_{p}=\frac{1}{2} \sum_{i=1}^{2}\left\{\sum_{n=1}^{N_{i}} \int_{P Z T_{n}} E_{p} z^{2}\left(\frac{\partial^{2} w_{p_{n}}}{\partial x^{2}}\right)^{2} d v\right. \\
\left.+\sum_{n=1}^{N_{i}} \int_{P Z T_{n}} z e_{31} E_{z_{n}} \frac{\partial^{2} w_{p_{n}}}{\partial x^{2}} d v+\frac{1}{2} \sum_{n=1}^{N_{i}} \int_{P Z T_{n}} E_{z_{n}} d_{n} d v\right\} ;
\end{gathered}
$$

wherein $z$ is the axis perpendicular to the piezoelectric surface, and $d_{n}$ is the electric displacement for the $n$th patch of piezoelectric layers. The last term in Eq. (2) is the electric energy stored in the piezoelectric materials. $E_{z_{n}}$ is electric field of the $n$th piezoelectric layer.

Considering $w_{j}$ as:

$$
w_{j}(x, t)=\sum_{i} \varphi_{i}^{j} q_{i}^{j}=\left(\boldsymbol{\Phi}^{j}\right)^{T} \mathbf{q}^{j} \quad j=1,2
$$

and substituting Eqs. (1) and (2) in Lagrange equations, the governing equations of motion are obtained as:

$$
\begin{gathered}
{\left[\begin{array}{cc}
M_{\theta \theta} & M_{\theta q} \\
M_{q \theta} & M_{q q}
\end{array}\right]\left\{\begin{array}{c}
\ddot{\boldsymbol{\theta}} \\
\ddot{\boldsymbol{q}}
\end{array}\right\}+\left[\begin{array}{cc}
C_{\theta \theta} & C_{\theta q} \\
C_{q \theta} & C_{q q}
\end{array}\right]\left\{\begin{array}{c}
\dot{\boldsymbol{\theta}} \\
\dot{\boldsymbol{q}}
\end{array}\right\}} \\
+\left[\begin{array}{cc}
0 & 0 \\
0 & K_{q q}
\end{array}\right]\left\{\begin{array}{c}
\boldsymbol{\theta} \\
\boldsymbol{q}
\end{array}\right\}=\left\{\begin{array}{c}
\tau \\
\left.-K_{\text {pelastelect }_{a}} v_{a}\right\}
\end{array}\right.
\end{gathered}
$$

Here, $\phi_{i}^{j}$ is a series of trial mode shapes satisfying the boundary conditions, and $q_{i}^{j}$ is thetime dependent generalized coordinate for the $j$ th appendage. Next, $\theta=[\theta 1 \theta 2 \theta 3]^{T}$, where $\theta_{i}$ is the $i$ th $(i=1,2,3)$ rotation angle of the satellite, namely around the $z_{1}, x_{2}$, and $y_{3}$ axes, respectively. The vector of generalized coordinates of the $j$ th appendage is $q=$ $\left[\left(q_{1}\right)^{T}\left(q_{2}\right)^{T}\right]^{T}$ and $q_{j}$, which is used in Rayleigh-Ritz technique. Further, $\boldsymbol{\tau}$ and $\boldsymbol{v}_{a}$ are the vectorscontroller input torques and actuator voltages, respectively. The parameters of the mass matrix are determined as:

$$
\begin{aligned}
& \left(M_{\theta \theta}\right)_{i j}= \\
& \operatorname{trace} \sum_{k=1}^{2} \frac{\partial T_{a_{k}}^{o}}{\partial \theta_{j}} \int_{\text {appendage } e_{k}} r_{a_{n}} r_{a_{n}}^{T} d m\left(\frac{\partial T_{a_{k}}^{o}}{\partial \theta_{i}}\right)^{T} \\
& + \text { trace } \sum_{k=1}^{2} \sum_{n=1}^{N_{k}} \frac{\partial T_{a_{k}}^{o}}{\partial \theta_{j}} \int_{P Z T_{n}} r_{a_{n}} r_{a_{n}}^{T} d m\left(\frac{\partial T_{a_{k}}^{o}}{\partial \theta_{i}}\right)^{T} \\
& + \text { trace } \frac{\partial T_{h}^{o}}{\partial \theta_{i}} \int_{h u b} r_{h} r_{h}^{T} d m\left(\frac{\partial T_{h}^{o}}{\partial \theta_{j}}\right)^{T} \\
& \left(M_{\theta q}\right)_{i j}=\left(M_{q \theta}\right)_{j i}=\text { trace } \sum_{k=1}^{2} \frac{\partial T_{a_{k}}^{o}}{\partial \theta_{j}} \\
& \int_{\text {appendage }_{k}} \frac{\partial \dot{r}_{a_{n}}}{\partial \dot{q}_{i}} r_{a_{n}}^{T} d m\left(T_{a_{k}}^{o}\right)^{T} \\
& +\operatorname{trace} \sum_{k=1}^{2} \sum_{n=1}^{N_{k}} \frac{\partial T_{a_{k}}^{o}}{\partial \theta_{j}} \int_{P Z T_{n}} \frac{\partial \dot{r}_{a_{n}}}{\partial \dot{q}_{i}} r_{a_{n}}^{T} d m\left(T_{a_{k}}^{o}\right)^{T} \text {; } \\
& \left(M_{q q}\right)_{i j}= \\
& \frac{1}{2} \operatorname{trace} \sum_{k=1}^{2} T_{a_{k}}^{o} \int_{\text {appendage }} \frac{\partial^{2}\left(\dot{r}_{a_{n}} \dot{r}_{a_{n}}^{T}\right)}{\partial \dot{q}_{i} \partial \dot{q}_{j}} d m\left(T_{a_{k}}^{o}\right)^{T}
\end{aligned}
$$




$$
+\frac{1}{2} \text { trace } \sum_{k=1}^{2} \sum_{n=1}^{N_{k}} T_{a_{k}}^{o} \int_{P Z T_{n}} \frac{\partial^{2}\left(\dot{r}_{a_{n}} \dot{r}_{a_{n}}^{T}\right)}{\partial \dot{q}_{i} \partial \dot{q}_{j}} d m\left(T_{a_{k}}^{o}\right)^{T} \text {. }
$$

where $T_{a i}^{o}$ and $T_{h}^{o}$ are the rotational/transitional matrix from the $i$ th appendage coordinate and hub frame to the base frame, respectively, and $r_{(\cdot)}$ is the position vector of the elementcoordinate $(\cdot)$. So $r_{h}$ is the position vector of an element in the hub coordinate. The matrixcentrifugal and Coriolis effects, $C$, in Eq. (4) is determined as: ${ }^{18}$

$$
\begin{aligned}
C_{i j} & =\sum_{k} c_{i j k} \dot{\xi} \\
c_{i j k} & =\frac{1}{2}\left(\frac{\partial M_{i j}}{\partial \xi_{k}}+\frac{\partial M_{i k}}{\partial \xi_{j}}-\frac{\partial M_{j k}}{\partial \xi_{i}}\right) ; \\
\zeta & =\left[\begin{array}{ll}
\theta^{\mathrm{T}} & q^{T}
\end{array}\right]^{T} .
\end{aligned}
$$

The stiffness matrix is $K_{q q}=\operatorname{diag}\left(K_{q q 1}, K_{q q 2}\right)$ and:

$$
\begin{aligned}
& K_{q q_{k}}=\int_{\text {appendage }_{k}} E I \frac{\partial^{2} \boldsymbol{\Phi}^{k}(x)}{\partial x^{2}} \frac{\partial^{2}\left(\boldsymbol{\Phi}^{k}(x)\right)^{T}}{\partial x^{2}} d x \\
&+\sum_{n=1}^{N_{k}} \int_{P Z T_{n}} E_{p} I_{p} \frac{\partial^{2} \boldsymbol{\Phi}^{k}(x)}{\partial x^{2}} \frac{\partial^{2}\left(\boldsymbol{\Phi}^{k}(x)\right)^{T}}{\partial x^{2}} d x \\
& \quad k=1,2 .
\end{aligned}
$$

where $I_{p}$ is the moment of inertia of the piezoelectric layers. The elastic-electric effect of the ppiezoelectric actuators is illustrated in $K_{\text {pelastelect }_{a}}$.

$$
K_{\text {pelastelect } a}=\left[\begin{array}{cc}
K_{\text {elastelect }_{a}}^{1} & 0 \\
0 & K_{\text {plastelect }_{a}}^{2}
\end{array}\right]
$$

where 0 is the zero matrix and

$$
K_{\text {plastelecta }}^{i}=\left[\begin{array}{llll}
K_{\text {pee }_{1}}^{i} & K_{\text {pee }_{2}}^{i} & \cdots & K_{\text {pee }_{N_{i}}}^{i}
\end{array}\right] .
$$

Here, $K_{\text {pee }_{j}}^{i}$ is the vector of the $j$ th column of the elasticelectric matrix and is defined as:

$$
K_{\text {pee }_{j}}^{i}=\frac{e_{31 j}}{h_{p_{j}}} \int_{P Z T_{j}} z \frac{\partial^{2} \boldsymbol{\Phi}^{i}}{\partial x^{2}} d v .
$$

Equation (4) can be rewritten as:

$$
M(\psi) \ddot{\psi}+C(\psi, \dot{\psi}) \dot{\psi}+K \psi=Y \rho=u
$$

where $u=\left[\tau^{T}\left(-K_{\text {pelastelecta }} v_{a}\right)^{T}\right]^{T}$ is the controller input, $\psi=\left[\boldsymbol{\theta}^{T} q^{T}\right]^{T}$, and $\rho$ is the parameters vector of the system.

\section{ROBUST CONTROLLER DESIGN}

If the bounds on the parameters of the system are known but these parameters are not exactly known, then the robust control scheme can be used. Considering the following controller vector: ${ }^{18}$

$$
u=\hat{M} \cdot\left[\ddot{\psi}_{d}-K_{D} \cdot \dot{\tilde{\psi}}-K_{P} \cdot \tilde{\psi}\right]+\hat{C} \cdot \dot{\psi}+\hat{K} \psi+u_{\circ}
$$

where $\hat{M}, \hat{C}$, and $\hat{K}$ represent nominal values vis-à-vis to parameter uncertainty of $M, C$, and $K$. Also, $\psi_{d}$ is the bounded, twice-differentiable desired vector of generalized coordinates, the tracking error is $\tilde{\psi}=\psi-\psi_{d}, u_{o}$ is an additional control input that will be defined later, and $K_{D}$ and $K_{p}$ are constant and positive definite diagonal matrices. Substituting the control input (12) into the dynamic model (11) gives the following error equation:

$$
M \cdot\left[\ddot{\psi}+K_{D} \cdot \dot{\tilde{\psi}}+K_{P} \cdot \tilde{\psi}\right]=Y \cdot \tilde{\rho}+u_{o}
$$

using the property of linearity of dynamic equation with respect to a set of constant physical parameters $\rho$.

Equation (13) can be rewritten in state space form as follows:

$$
\dot{\xi}=\left[\begin{array}{cc}
0 & I \\
-K_{P} & -K_{D}
\end{array}\right] \xi+\left[\begin{array}{c}
0 \\
M^{-1}
\end{array}\right]\left(Y(\cdot) \tilde{\rho}+u_{o}\right) .
$$

Here, $\xi=\left[\begin{array}{ll}\xi_{1}^{T} & \xi_{2}^{T}\end{array}\right]^{T}$ is the state vector, and $\xi_{1}=\tilde{\psi}$ and $\xi_{2}=\dot{\tilde{\psi}}$ are the state variables.

The compact form of Eq. (14) is:

$$
\dot{\xi}=A \xi+B M^{-1} u_{o}(\xi)+B e(\xi) .
$$

Here,

$A=\left[\begin{array}{cc}0 & I \\ -K_{P} & -K_{D}\end{array}\right] ; B=\left[\begin{array}{l}0 \\ I\end{array}\right] ; e(\xi)=M^{-1} Y(\cdot) \tilde{\rho}$.

Consider the following positive definite Lyapunov function: ${ }^{18}$

$$
V=\xi^{T} P \xi
$$

Here, $P$ is a symmetric positive definite matrix satisfying $A^{T} P+P A=Q$, with $Q$ being symmetric and positive definite too. Tacking the time derivative of $V$ along the trajectories of the error system (13) yields:

$$
\dot{V}=-\xi^{T} Q \xi+2 \xi^{T} P B M^{-1}\left(Y(\cdot) \tilde{\rho}+u_{o}\right) .
$$

From Eq. (18) it follows that

$\dot{V} \leq-\xi^{T} Q \xi+2\left\|\xi^{T} P B\right\|\left\|M^{-1} Y(\cdot) \tilde{\rho}\right\|+2 \xi^{T} P B M^{-1} u_{o}$.

We now make the assumptions that there exists a known function $\beta(\cdot): R^{2 n} \times R \rightarrow R^{l}$ and a constant vector $a * \in R^{l}$ such that:

$$
\begin{aligned}
\beta_{i}(\xi, t) & \geq 0 ; \\
\alpha_{i}^{*} & \geq 0 \quad 1 \leq i \leq l ; \\
\left\|M^{-1} Y(\cdot) \tilde{\rho}\right\| & \leq \beta^{T}(\xi, t) \alpha^{*} .
\end{aligned}
$$

for all $(\xi, t) \in R^{2 n} \times R$.

From properties of the dynamic model described above, a possible choice for $\beta^{T}(\xi, t) \alpha^{*}$ is:

$$
\beta^{T}(\xi, t) \alpha^{*}=\alpha_{1}^{*}+\alpha_{2}^{*}\|\psi\|+\alpha_{3}^{*}\|\dot{\psi}\|+\alpha_{4}^{*}\|\dot{\psi}\|^{2} .
$$

Choosing the additional control input $u_{o}$ as:

$$
u_{o}=-\frac{\left(\beta^{T}(\xi, t) \alpha^{*}\right)^{2} \lambda}{B^{T} P \xi}
$$




\begin{tabular}{l}
\hline \hline Table 1. Characteristics of the piezoelectric layers and flexible appendages. \\
\begin{tabular}{||c|c|}
\hline Piezoelectric layer & Flexible appendage \\
\hline Modulus, $E_{p}=2 \times 10^{9} \mathrm{~N}^{1} \mathrm{~m}^{2}$ & Modulus, $E=76 \times 10^{9} \mathrm{~N} / \mathrm{m}^{2}$ \\
\hline Piezoelectric constant, & \\
$d_{p}=22 \times 10^{-12} \mathrm{mV}-1$ & Length, $L=5 \mathrm{~m}$ \\
\hline Thickness, $h_{p}=1 \mathrm{~mm}$ & Thickness, $h=8 \mathrm{~mm}$ \\
\hline Density, $\rho_{p}=1800 \mathrm{~kg} / \mathrm{m}^{3}$ & Width, $b=0.5 \mathrm{~m}$ \\
\hline & Density, $\rho=2840 \mathrm{~kg} / \mathrm{m}^{3}$ \\
\hline & Radius of hub, $l=1 \mathrm{~m}$ \\
\hline
\end{tabular}
\end{tabular}

and using Eq. (20), Eq. (19) can be rewritten as:

$$
\begin{aligned}
& \dot{V} \leq-\xi^{T} Q \xi \\
& +\frac{2}{\varepsilon}\left\|\xi^{T} P B\right\| \beta^{T}(\xi, t) \alpha^{*}\left(\varepsilon-\left\|\xi^{T} P B\right\| \beta^{T}(\xi, t) \alpha^{*}\right) .
\end{aligned}
$$

Here, we have used the fact that $\lambda_{\min }\left(M^{-1}\right)=1 / \lambda_{M}$, wherein $\epsilon>0, \lambda>\lambda_{M}$, and $\lambda_{M}$ is the greatest eigenvalue of the mass matrix, $M$. Therefore, as long as the following inequality is verified:

$$
\left\|\xi^{T} P B\right\| \beta^{T}(\xi, t) \alpha^{*} \geq \epsilon .
$$

We get:

$$
\dot{V}<-\xi^{T} Q \xi .
$$

This inequality is strict; indeed, we can show that if $\xi=0$ then:

$$
\dot{V} \leq-\xi^{T} Q \xi+2 \epsilon .
$$

This shows the state $\xi$ is globally ultimately uniformly bounded with respect to a compact set around the origin $\xi=0$.

\section{SIMULATION RESULTS}

In order to numerically solve the governing equation of motion and observe the response of the closed-loop control system, the governing equation of motion is solved using Newmark integration. The piezoelectric layers and satellite parameters are given in Table 1.

We consider large angles trajectory tracking around the $x_{2}$, $y_{3}$, and $z_{1}$ axes as $\pi / 3, \pi / 4$, and $\pi / 6$, respectively. The desired trajectory angles of the satellite have been defined as the following function:

$$
\theta_{d}=\left[6\left(\frac{t}{t_{f}}\right)^{5}-15\left(\frac{t}{t_{f}}\right)^{4}+10\left(\frac{t}{t_{f}}\right)^{3}\right]\left(\theta_{f}-\theta_{0}\right)+\theta_{0} .
$$

Herein $t_{f}$ is the total time needed to complete a manoeuvre, and $\theta_{f}$ and $\theta_{0}$ are the initial and final angular positions, respectively.

In order to show high effectiveness and performances of the robust controller, nonzero initial conditions, which lead to greater amplitude appendages vibrations, are considered. The controller gains have been selected as: $K_{p}=$ diag[[500 500500250200100250200100$]$ and $K_{D}=$ diag[$\left[\begin{array}{lllllllll}460 & 460 & 460 & 100 & 80 & 80 & 100 & 80 & 80\end{array}\right]$. Six pairs of piezoelectric layers are considered to be attached to both the appendages. The length of all piezoelectric layers is same, $0.5 \mathrm{~m}$. The piezoelectric actuators are located at the base, mid$\mathrm{dle}$, and the end of the appendages. Figure 2 shows the angular trajectory tracking of the manoeuvring satellite around $x_{2}, y_{3}$, and $z_{1}$ axes and its desired one. It can be seen that the desired trajectory is effectively tracked by satellite. Figure 3 illustrates the tip deflection of the right appendage when voltage is applied to the piezoelectric actuators and without applying voltage to them. This figure shows that this robust controller can (a)

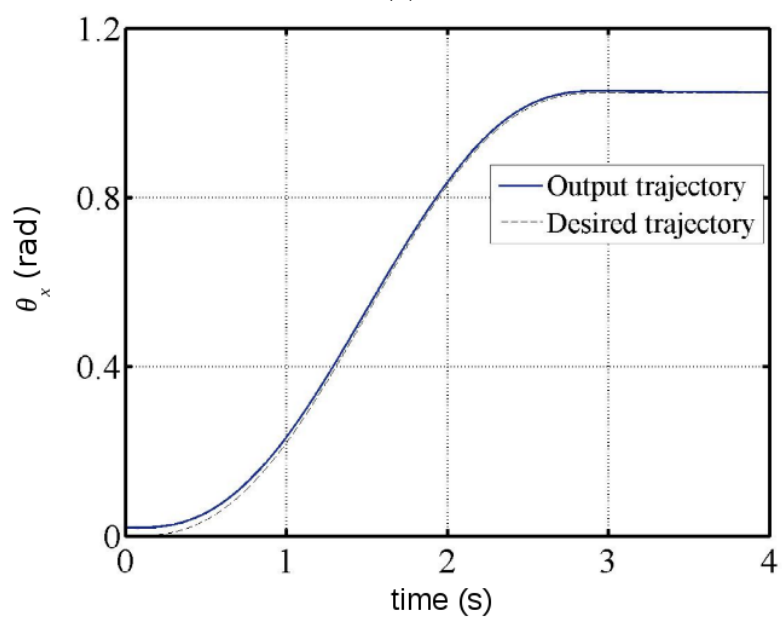

(b)

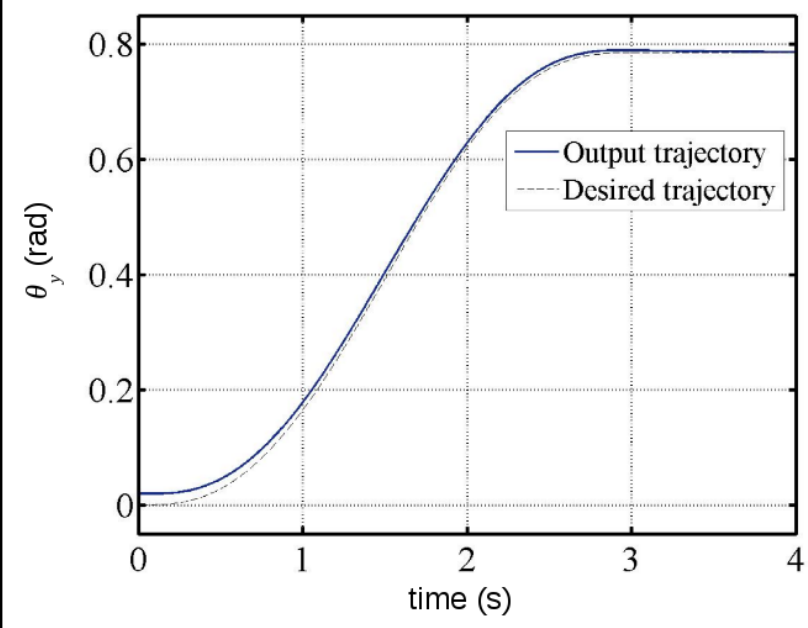

(c)

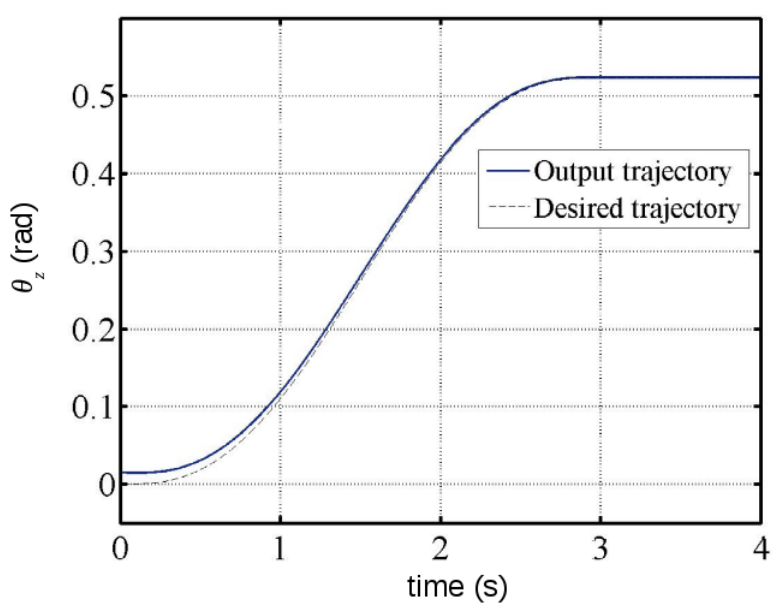

Figure 2. Rotational trajectory tracking of the satellite. (a) around $x_{2}$ axis, (b) around $y_{3}$ axis, (c) around $z_{1}$ axis.

suppress the appendage vibrations rapidly by applying voltage to piezoelectric actuators. Since there is a symmetry in the system, the tip deflection of the left appendage is similar to Fig. 3. Figure 4 compares the generalized coordinates of the controlled system with uncontrolled one. This figure shows that all three generalized coordinates of the right appendage converge to zero by applying the voltages to the piezoelectric actuators. This means that the vibrations of this appendage have been damped and converge to zero, too. The simulation 


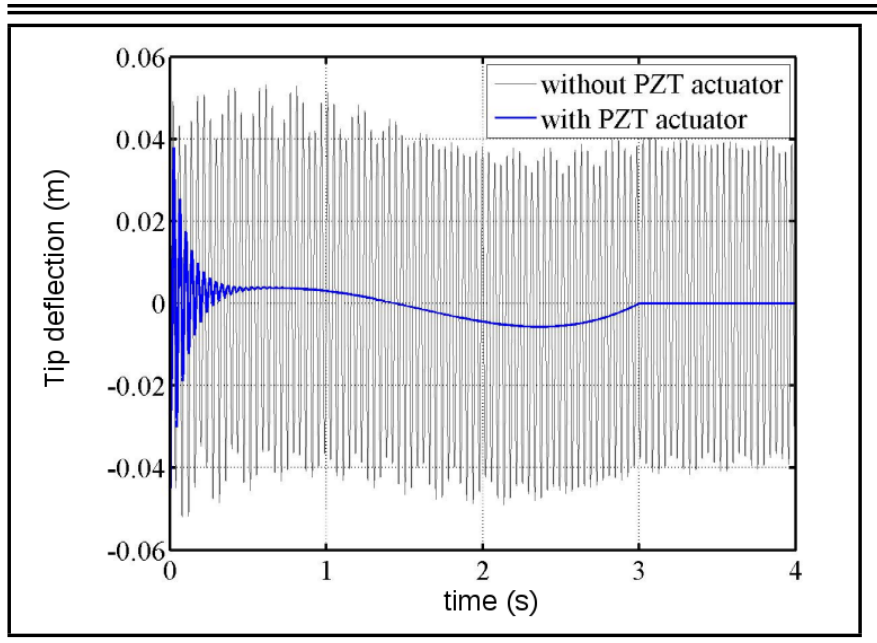

Figure 3. Tip deflection of the right appendage with and without piezoelectric actuators.

results for the left appendage are similar to the right one. Voltages which are applied to the actuators are shown in Fig. 6. According to Fig. 6, the controller applies the admissible voltages to the actuators. ${ }^{19}$ The mid vibrations of the right appendage have been shown in Fig. 5. The simulation results show the high performance and capability of the controller.

\section{CONCLUSION}

A robust control scheme was used to control the manoeuvre and vibration of a flexible satellite. A satellite with two flexible appendages was considered as a central hub. Piezoelectric layers attached to appendages were used as actuators to suppress the vibration of the appendages. Further, nonlinear governing equations of motion were derived. The robust control scheme was defined and applied to the system. Finally, the system was simulated and the simulation results showed the high capability of this robust controller.

\section{REFERENCES}

1 Song, G. and Kotejoshyer, B. Vibration reduction of flexible structures during slew operations, International Journal of Acoustics and Vibration, 7 (2), 105-109, (2002).

2 Ge, S.S., Lee, T.H., Hong, F., and Goh, C.H. Energybased robust controller design for flexible spacecraft, Journal of Control Theory and Applications, 2, 27-34, (2004). https://dx.doi.org/10.1007/s11768-004-0020-z

${ }^{3} \mathrm{Hu}, \mathrm{Q}$. Robust adaptive sliding mode attitude maneuvering and vibration damping of three-axis stabilized flexible spacecraft with actuator saturation limits, Nonlinear Dynamics, 55, 301-321, (2009). https://dx.doi.org/10.1007/s11071-008-9363-1

4 Erdong, J., and Zhaowei, S. Passivity-based control for a flexible spacecraft in the presence of disturbances, International Journal of Nonlinear Mechanics, 45, 348-356, (2010). https://dx.doi.org/10.1016/j.ijnonlinmec.2009.12.008

5 Azadi, E., Eghtesad, M., Fazelzadeh, S. A., and Azadi, M. Vibration suppression of smart nonlinear flexible appendages of a rotating satellite by using hybrid adaptive sliding mode/Lyapunov control, Jour- (a)

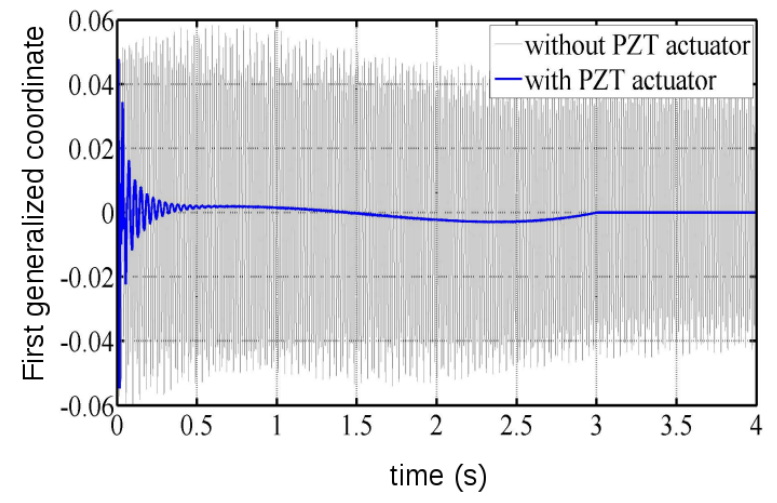

(b)

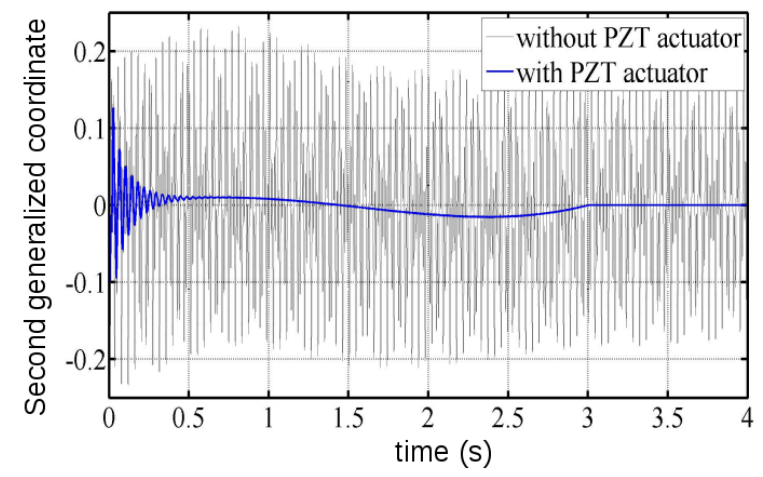

(c)

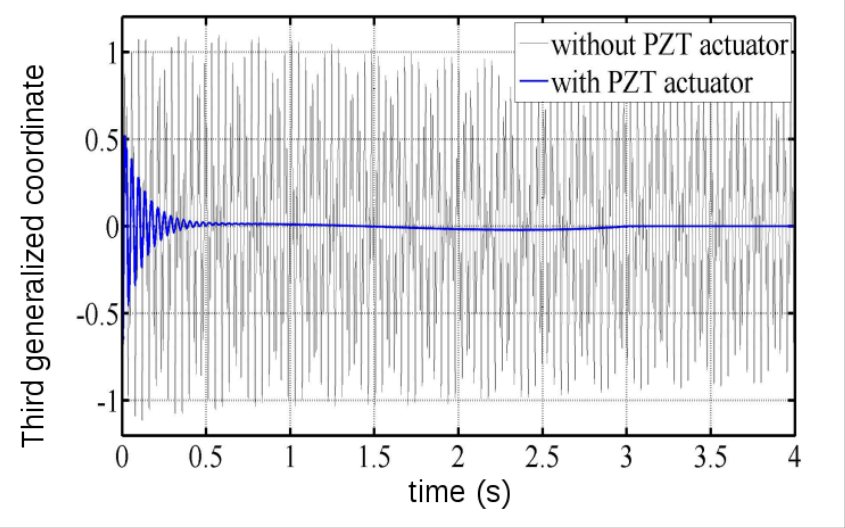

Figure 4. Time history of the right appendage generalized coordinates. (a) first generalized coordinate, (b) second generalized coordinate, (c) third generalized coordinate.

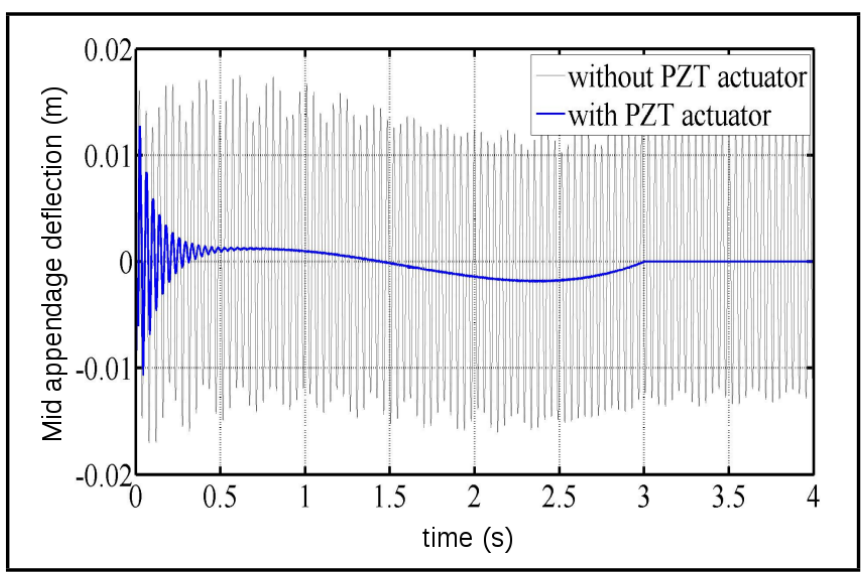

Figure 5. Mid deflection of the right appendage with and without piezoelectric actuators. 


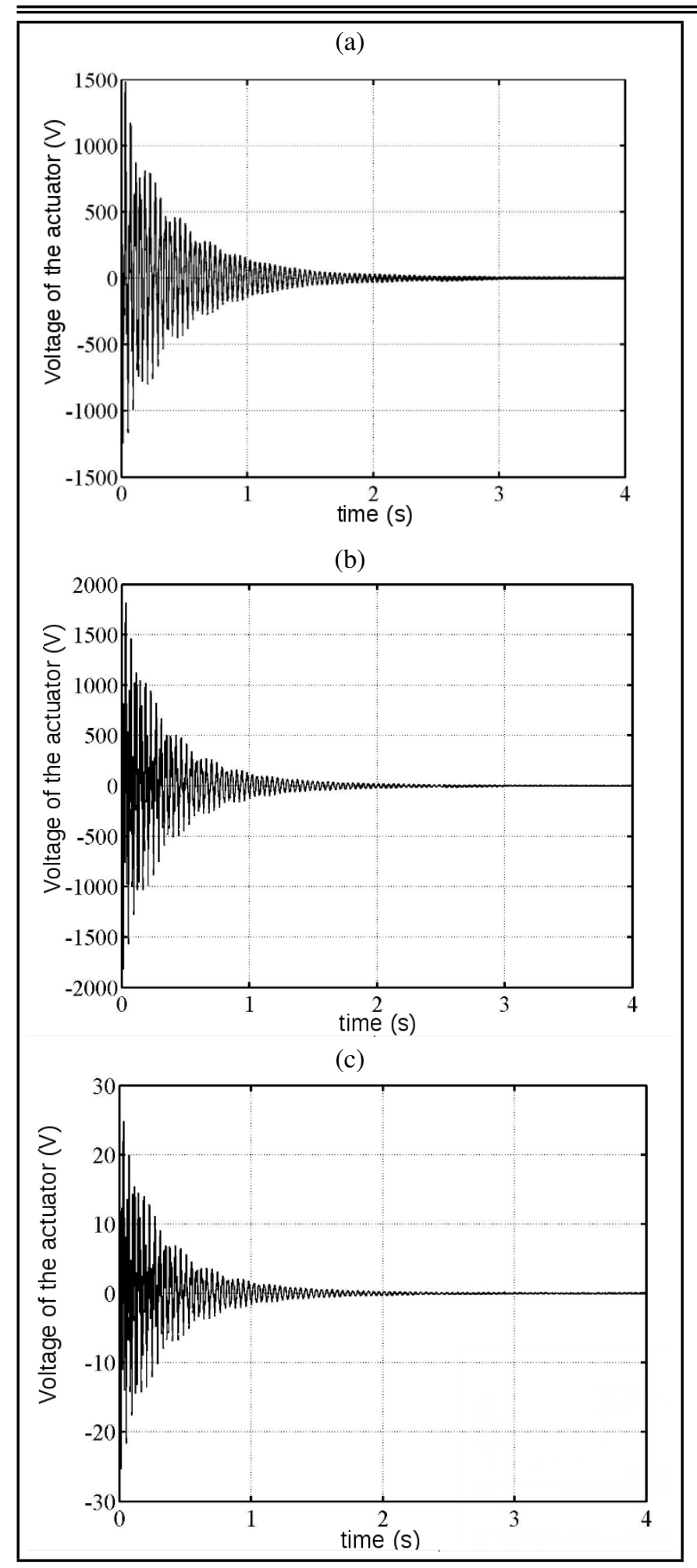

Figure 6. Input voltages of piezoelectric actuators bonded to the right appendage. (a) first piezoelectric layer, (b) second piezoelectric layer, (c) third piezoelectric layer.

nal of Vibration and Control, 19 (7), 975-991, (2013). https://dx.doi.org/10.1177/1077546312439913

6 Azadi, E., Fazelzadeh, S. A., Eghtesad, M., and Azadi, M. Active vibration suppression and maneuver control of an orbiting smart flexible satellite, IJST, Transactions of Mechanical Engineering, 38 (M1), 119-133, (2014).

$7 \mathrm{Hu}$, Q. and Ma, G. Variable structure control and active vibration suppression of flexible spacecraft during attitude maneuver, Aerospace Science and Technology, 9, 307-317, (2005). https://dx.doi.org/10.1016/j.ast.2005.02.001

${ }^{8} \mathrm{Hu}$, Q. A composite control scheme for attitude maneuvering and elastic mode stabilization of flexible spacecraft with measurable output feedback, Journal of Aerospace and Technology, 13, 81-91, (2009). https://dx.doi.org/10.1016/j.ast.2007.06.007

9 Neto, M. A., Ambrsio, J. A., Roseiro, L. M., Amaro, A., and Vasques, C. M. A. Active vibration control of spatial flexible multibody systems, Multibody System Dynamics, 30 (1), 13-35, (2013). https://dx.doi.org/10.1007/s11044013-9341-3

10 Sales, T. P., Rade, D. A., and de Souza. L. C. G, Passive vibration control of flexible spacecraft using shunted piezoelectric transducers, Aerospace Science and Technology, 29 (1), 403-412, (2013). https://dx.doi.org/10.1016/j.ast.2013.05.001

$11 \mathrm{Hu}, \mathrm{Q}$. Semi-globally input-to-state stable controller design for flexible spacecraft attitude stabilization under bounded disturbances, Acta Astronautica, 66, 567-576, (2010). https://dx.doi.org/10.1016/j.actaastro.2009.07.012

12 Jiang, Y., Hu, Q., and Ma, G. Adaptive backstepping fault-tolerant control for flexible spacecraft with unknown bounded disturbances and actuator failures, ISA Transactions Journal, 49, 57-69, (2010). https://dx.doi.org/10.1016/j.isatra.2009.08.003

13 Xin, M., and Pan, H. Nonlinear optimal control of spacecraft approaching a tumbling target, Aerospace Science and Technology, 15 (2), 79-89, (2011). https://dx.doi.org/10.1016/j.ast.2010.05.009

14 Azadi, M., Fazelzadeh, S.A., Eghtesad, M., and Azadi, E. Vibration suppression and adaptive-robust control of a smart flexible satellite with three axes maneuvering, Acta Astronautica, 69, 307-322, (2011). https://dx.doi.org/10.1016/j.actaastro.2011.04.001

15 Verbin, D., and Lappas V.J. Rapid rotational maneuvering of rigid satellites with hybrid actuators configuration, Journal of Guidance, Control, and Dynamics, 36 (2), 532-547, (2013). https://dx.doi.org/10.2514/1.56405

16 Verbin, D., and Lappas V.J. Rapid rotational maneuvering of rigid satellites with reaction wheels, Journal of Guidance, Control, and Dynamics, 36 (5), 1538-1544, (2013). https://dx.doi.org/10.2514/1.58155

17 Huang, X., Yan, Y., and Zhou, Y. Dynamics and control of spacecraft hovering using the geomagnetic Lorentz force, Advances in Space Research, 53 (3), 518-531, (2014). https://dx.doi.org/10.1016/j.asr.2013.11.040

18 Canudas, C., Siciliano, B., and Bastin, G. Theory of Robot Control, Springer-Verlag, (1997).

$19 \mathrm{Li}$, Z., and Bainum, P. M. Vibration control of flexible spacecraft integrating a momentum exchange controller and a distributed piezoelectric actuator, Journal of Sound and vibration, 177 (4), 539-553, (1994). https://dx.doi.org/10.1006/jsvi.1994.1450 